\title{
Letter
}

\section{A Case Report: A Rare Case of Multiple Chest Wall Schwannomas Associated with Uterine Leiomyoma and Infertility}

\author{
Kawkabani Nadine", ", Darwish Rula ${ }^{1}$, Boustros Omar², Bejjani Simon ${ }^{2}$, El Achkar Nancy ${ }^{3}$, \\ Abou Khalil Bassam ${ }^{4}$ \\ ${ }^{1}$ Cardiac Surgery Anesthesia Department, Saint Georges Hospital University Medical Center, Beirut, Lebanon \\ ${ }^{2}$ Cardio-Thoracic Surgery Department, Saint Georges Hospital University Medical Center-University of Balamand, Beirut, Lebanon \\ ${ }^{3}$ General Anesthesia Department, Saint Georges Hospital University Medical Center-University of Balamand, Beirut, Lebanon \\ ${ }^{4}$ Cardio-Thoracic Surgery Department, Saint Georges Hospital University Medical Center, Beirut, Lebanon
}

\section{Email address:}

nadine_kaoukabani@yahoo.com (K. Nadine)

${ }^{*}$ Corresponding author

\section{To cite this article:}

Kawkabani Nadine, Darwish Rula, Boustros Omar, Bejjani Simon, El Achkar Nancy, Abou Khalil Bassam. A Case Report: A Rare Case of Multiple Chest Wall Schwannomas Associated with Uterine Leiomyoma and Infertility. International Journal of Cardiovascular and Thoracic Surgery. Vol. 4, No. 1, 2018, pp. 1-4. doi: 10.11648/j.ijcts.20180401.11

Received: November 12, 2017; Accepted: December 14, 2017; Published: January 8, 2018

\begin{abstract}
Schwannomatosis is a tumor predisposition syndrome characterized by development of multiple schwannomas that are slow-growing encapsulating neurogenic tumors. It is a recurrent condition and patients lack usually the classic cutaneous stigmata, vestibular schwannomas or brain parenchyma tumors. Many papers have incriminated the mutation of the SMARCB1 gene in this disease which has been also observed in cases of uterine leiomyoma. This report describes a rare case of multiple chest wall schwannomas associated to a uterine fibroma. A genetic study done did not show any SMARCB1 gene mutation. Subsequently a further genetic counselling and a whole exome sequencing were recommended especially that the patient underwent many trials of failed IVF and two miscarriages despite resection of all the tumors.
\end{abstract}

Keywords: Schwannomatosis, Leiomyoma, Infertility, Genetic Mutation

\section{Introduction}

Schwannomas, also known as neurilemomas are slow-growing encapsulated neurogenic tumors. Chest wall schwannomas are rare and usually asymptomatic growing towards the pleural space in the posterior mediastinum. Most neural tumors of the chest arise in the mediastinum. Fewer than $10 \%$ of originate peripherally from intercostal nerves. They typically occur in patients between the second and fith decade [1] [2]. On the other hand malignant schwannomas are high grade sarcomas that most commonly arise in young and middle aged adults. They are fast growing mass mainly in the trunk and proximal parts of the extremities [1] [2]. Shwannomatosis is defined as multiple schwannomas in patients who has none of the stigmata of any of the neurofibromatosis [3]. It has been associated in some cases with uterine fibroma and many papers have shown the incrimination of a genetic mutation on chromosome 22 [3]. The following report describes a case of multiple chest schwannomas associated to a uterine fibroma and primary infertility.

\section{Case Report}

A 36 year old female known to be smoker, dyslipidemic and asthmatic was admitted to our institution for multiple chest wall mass resection.

The patient started complaining of progressive dyspnea and 
dry cough two months earlier. On physical exam, she had expiratory wheezes on the left upper lung.

A chest $\mathrm{X}$ ray revealed the presence of three round lesions of the left chest wall.

A CT scan of the chest showed a mass at the 7th intercostal space $(2 * 2.3 \mathrm{~cm})$ and two heterogeneous lesions at the cardio-phrenic angle $(4 * 2.3 \mathrm{~cm})$ in the para cardiac region (sitting on the right ventricule). No signs of costal erosions were noticed. (Figure 1).

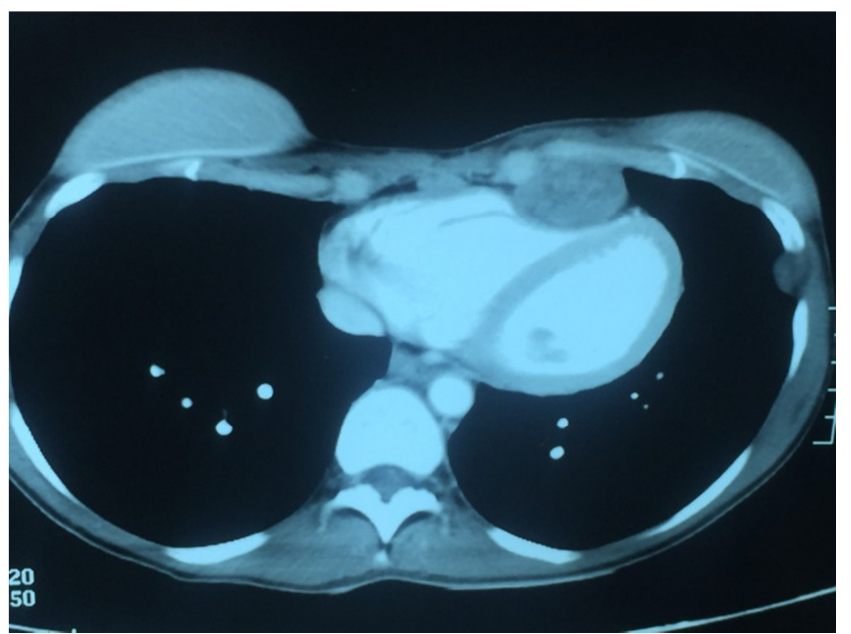

Figure 1. CT scan of the chest preoperatively showing the lesions in the paracardiac region sitting on the right ventriule $(4 * 2.3 \mathrm{~cm})$ and at the $7^{\text {th }}$ intercostal space $(2 * 2.3 \mathrm{~cm})$.

A biopsy under ultrasound guidance for the intercostal lesion revealed that it was of neural origin most probably schwannoma. PET scan showed minimal activity of the different lesions.

Surgical resection of the lesions was scheduled. A preoperative spirometry revealed a $\mathrm{FEV} 1 / \mathrm{VC}$ of $78 \%$ and a FEV1 of $2.51 / \mathrm{min}$. All the laboratory studies and arterial blood gas were within normal range.

The patient was induced and intubated after the insertion of a thoracic epidural at the level T6-T7 for post- operative pain control.

A left anterolateral thoracotomy was done. Two large masses sitting on the right and left ventricle with compression and one lateral mass of the chest wall were entirely resected. No unusual adhesions were encountered. The intraoperative frozen biopsy showed neural tumors suggestive of schwannomas.

At the end of surgery, the patient was extubated and transferred to the intensive care unit (ICU) for observation. The postoperative course was uneventful. She was transferred to the floor the next day and discharged from the hospital 5 days after surgery.

The final pathology report confirmed the diagnosis of schwannomas without any signs of malignancy. Five months later the patient was doing well. A chest $\mathrm{x}$ ray and a CT scan showed no signs of reccurence. (figure 2).

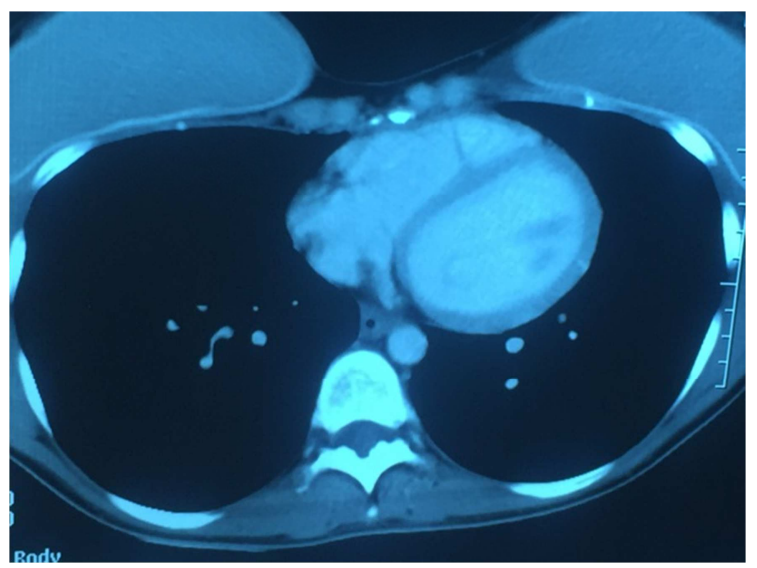

Figure 2. CT scan of the chest five months post operatively shows no signs of recurrence.

One year following the thoracic surgery, the patient was operated for a uterine fibroid resection (leiomyoma). She underwent afterwards many failed in vitro fecondation (IVF) and two miscarriages.

She was advised to consult for a genetic opinion especially that she developed both chest wall shwannomas and uterine leiomyoma. Blood studies were done in order to define any gene mutation. Results showed no relevant variant nor deletion or duplication of the SMARCB1 gene.

\section{Discussion}

Schwannomas, also known as neurilemomas or neurinomas are slow -growing encapsulating neurogenic tumors [1].

Chest wall schwannomas are rare and usually found as asymptomatic solitary lesions growing towards the pleural space in the posterior mediastinum. They arise from spinal nerve roots and intercostal nerves and typically occur in patients between the second and fith decades $.10 \%$ of the neural tumors of the chest originate peripherally from intercostal nerves. $95 \%$ of the neurogenic tumors are nerve sheath tumors and of these $75 \%$ are neurilenomas [2].

Schwannomatosis is defined as multiple schwannomas in patients who have none of the stigmata of any of the neurofibromatosis. It is a reccuring condition in patients with multiple schwannomas. Patients lack usually the classic cutaneous stigmata, vestibular schwannomas, neurofibromas or tumors of the brain parenchyma [3].

In this case report, the 36 year old female patient developed multiple lesions of neural origin at the $7^{\text {th }}$ intercostal space and the left cardiophrenic angle that were defined as schwannomas by the pathology exam. No cutaneous signs nor brain tumors were present. To note that she was operated also for uterine fibroid resection one year after her thoracotomy.

Many studies have shown that patients with schwannomatosis have an abnormality on the chromosome 22 . Because this entity is rare, its inheritance pattern was unknown but recent studies found in 2007 a genetic mutation of SMARCB1 a tumor suppression gene on chromosome 22 that was responsible of this disease [4].

In subsequent studies, it became clear that the gene is 
involved in about $50 \%$ of the familial cases but no more than $10 \%$ of the sporadic cases [5]. Most but not all germline mutations in the SMARCB1 gene are missense or splice site mutations. They result in the synthesis of SMARCB1 proteins with altered activity [4]. Besides schwannomas, other tumor types have been also reported to develop at a low frequency in schwannomatosis patients notably vestibular schwannomas, meningiomas and leiomyomas [6] [7].

A study done by Hulsebos et al reported the occurrence of a leiomyoma of the uterine cervix in a familial schwannomatosis patient. They found that leiomyomas displayed the genetic and immunohistochemical characteristics of a SMARCB1 mutation positive in schwannomatosis tumors, widening further the spectrum of benign tumors associated with germline SMARCB1 mutation They suggested that in cases in which female patients develop uterine and extrauterine pelvic leiomyomas and schwannomas it might be of value to determine whether they are carriers of a germline SMARCB1 mutation [8].

Few cases in the literature reported also the occurrence of leiomyomas and schwannomas. Dane et al described a case in which an association of a vaginal schwannomas and a uterine leiomyoma was present [9]

Gorlin and Koutlas presented a large family with multiple schwannomas including cervical schwannomas in one patient and cervical polyps in another Multiple nevi did not manifest in all affected individuals. Multiple vaginal leiomyomas were also described. They concluded that it would be of interest to determine the SMARCB1 mutation status of the patients in this family [10].

In the last few years, clinical findings in the patients with schwannomatosis have been widened. Currently the spectrum of associated tumors include meningiomas and leiomyomas (benign tumors). However to date, malignant tumors have been reported only in very few cases that described malignant peripheral sheath tumors. Paganini et al reported for the first time a case in which the association of a malignant tumor with smooth muscle differentiation to schwannomatosis was observed. Therefore they recommended that clinicians should be aware that a constitutional SMARCB1 mutation that usually predisposes to nerve sheath tumors may also predispose to aggressive neoplasms throughout life with an unexpected spectrum [3].

This patient was operated for both schwannomas and leiomyomas. No similar cases were described in her family. To note that she underwent also many failed trials of IVF and two miscarriages. She underwent a genetic study in order to determine if any mutation was positive. The exam showed no relevant variant neither large deletion or duplication of the SMARCB1 gene. A genetic counselling and a somatic study were recommended by the genetic center.

The risk of malignancy in a nerve sheath tumor is very small (2-5\%) unless there is a history of Von Ricklenhausen's disease or radiation exposure. In such cases the risk increases to $10-20 \%$. Nerve sheath tumors can produce intercostal nerve irritation, rib displacement and bone erosion owing to pressure effect. It is often impossible to establish benign or malignant nature of these tumors before surgery. However, in certain cases the computed tomography scan can demonstrate central areas of low attenuation consistent with necrosis, hemorrhage or cystic degeneration which are suggestive of malignancy. Benign tumors can produce thickening and scalloping of the adjacent ribs but are generally free from it. The presence of unusual adherence indicates malignancy. Therefore a wide surgical resection remains the cornerstone of treatment and requires the cooperation of thoracic, plastic and neurosurgeons is important. [2, 11, 12, 13, 14, 15]

In this case, all the tumors were completely resected. No signs of malignancy were found on the pathologic study. A two years follow up showed no recurrence

\section{Conclusion}

The authors describe in this report a sporadic case of multiple chest wall schwannomas associated with a uterine leiomyma. A genetic study revealed no mutation of the SMARCB1 gene which is usually present in such cases especially the familial ones.

To note that the patient underwent also many failed IVF and two miscarriages despite resecting all these masses. The future plan is to undergo further genetic counselling for a wider genetic exam such as whole exome sequencing to test for a differential genetic diagnosis and search for any mutation that can be responsible for such lesions and infertility.

\section{References}

[1] Ilgan S, Dikmen E, çetinkanat CG, Dakak M and Güngor A. Schwannomatosis of the chest wall: FDG PET findings. Molecular imaging and radionuclide therapy, 2014; 23 (2): 64-66.

[2] Anderson BO, Burt ME. Chest wall neoplasms and their management. Ann thorac Surg. 1994; 58: 1774-1781.

[3] Paganini I, Sestini R, Cacciatore M, Capane G, Candita L, Parolello C, Sbaraglia M, Deitos AP, Rossi S, Papi L. Broadening the spectrum of SMARCB1- associated malignant tumors: a case of uterine leiomyosarcoma in a patient with schwannomatosis. Human pathology. 2015; 46: 1226-1231.

[4] Hulsebos TJM, plompAS, WoltermanRA et al. Germline mutation of IN1/SMARCB1 in familial schwannomatosis. Am J Hum Genet. 2007; 80: 805-810.

[5] Plotkin SR. Blakely JO, Evans DG et al. Update from the 2011 International Schwannomatosis workshop from genetics to diagnostic criteria. Am J Med Genet A. 2013; 161: 405-416.

[6] Smith MJ, KulkarniA, RustadC et al. Vestibular schwannomas occur in schwannomatosis and should not be consideredan exclusion criteria for clinical diagnosis. Am JMed Genet A. 2012; 158A: 215-218.

[7] Hulsebos TJM, Kenter S, Siebers-Renelt U, Hans V, Wesseling P, Flucke U. SMARCB1 involvement in the development of leiomyoma in a patient with schwannomatosis. Am J Surg Pathol. 2014; 38 (3): 421-425. 
[8] Bazzi C, SestiniR, ProvenzanoA et al. Shwannomatosis associatd with multiple meningiomas due $t$ a familial SMARCB1 mutation. Neurogenetics. 2001; 11: 73-80.

[9] Dane B, Danec, Basaran S etol. Vaginal schwannoma in a case with uterine Myoma. Ann Diagn Pathol. 2010; 14: 137-139.

[10] Gorlin RJ, Koutlas IG. Multiple Schwannomas, multiple nevi and multiple vaginal leiomyomas: a new dominant syndrome. Am J Med. Genet 1998; 78: 76-81.

[11] McClenethanJH, BloomRJ. Peripheral tumors of the intercostal nerves. AnnThoracSurg. 2004; 78: 713-714.

[12] AthanassiadiK, KalavrouziotisG, RondogianniD, LoutsidisA, HatzimichalisA, Bellenis I. Primary chest wall tumors: early and long term results of surgical treatment. Eur $\mathrm{J}$ cardiothorac surg. 2001; 19: 589-593.
[13] Sebanathan S, Salama FD, Morgan WE, Harvey JA. Primary chest wall tumors. Ann thorac surg 2004; 78: 713-714.

[14] Bousamra M. Neurogenic tumors of the mediastinumin: Pearson FG, Cooper JL, DeslauriersJ, Ginsberg RJ, Heibert CA, Patterson GA, Urshel HC, editors. Thoracic surgery. $2^{\text {nd }}$ ed, Philadelphia, PA: Churchill living-stone; 2002: 1732-1738.

[15] Pandiyan MS, Kavunkal AM, Cherian VK, Christopher DJ. Chest wall mass with double pathology. European journal of cardio -thoracic surgery. 2006; 29: 625-626.

[16] Tateishi U, Gladish GW, Kusumoto M, Hasegawa T, Yokoyama R, Tsuchiya R, Moriyama N. Chest wall tumors: Radiologic findings and pathologic correlation. Part 1. Benign tumors. Radiographics. 2003; 23: 1477-1490. 